ACCEPTED MANUSCRIPT

\title{
A measurable physical theory of hyper-correlations beyond quantum mechanics
}

To cite this article before publication: Tristan Farrow et al 2020 Phys. Scr. in press https://doi.org/10.1088/1402-4896/abc5ed

\section{Manuscript version: Accepted Manuscript}

Accepted Manuscript is "the version of the article accepted for publication including all changes made as a result of the peer review process, and which may also include the addition to the article by IOP Publishing of a header, an article ID, a cover sheet and/or an 'Accepted

Manuscript' watermark, but excluding any other editing, typesetting or other changes made by IOP Publishing and/or its licensors"

This Accepted Manuscript is @ 2020 IOP Publishing Ltd.

During the embargo period (the 12 month period from the publication of the Version of Record of this article), the Accepted Manuscript is fully protected by copyright and cannot be reused or reposted elsewhere.

As the Version of Record of this article is going to be / has been published on a subscription basis, this Accepted Manuscript is available for reuse under a CC BY-NC-ND 3.0 licence after the 12 month embargo period.

After the embargo period, everyone is permitted to use copy and redistribute this article for non-commercial purposes only, provided that they adhere to all the terms of the licence https://creativecommons.org/licences/by-nc-nd/3.0

Although reasonable endeavours have been taken to obtain all necessary permissions from third parties to include their copyrighted content within this article, their full citation and copyright line may not be present in this Accepted Manuscript version. Before using any content from this article, please refer to the Version of Record on IOPscience once published for full citation and copyright details, as permissions will likely be required. All third party content is fully copyright protected, unless specifically stated otherwise in the figure caption in the Version of Record.

View the article online for updates and enhancements. 


\title{
A measurable physical theory of hyper-correlations beyond quantum mechanics
}

\author{
Tristan Farrow, ${ }^{1,2}$ Vlatko Vedral, ${ }^{1,2}$ and Wonmin Son $^{3, *}$ \\ ${ }^{1}$ Centre for Quantum Technologies, National University of Singapore, 3 Science Drive 2, Singapore 117543 \\ ${ }^{2}$ Department of Physics, University of Oxford, Parks Road, Oxford OX1 3PU, UK \\ ${ }^{3}$ Department of physics, Sogang University, Mapo-gu, Shinsu-dong, Seoul 121-742, Korea
}

\begin{abstract}
A unique characteristic of quantum mechanics is entanglement describing correlations between particles irrespective of their locations. This property, called non-locality, has no classical analogue Over the past few years, quantum physicists have reached a consensus that we lack a physical theory to account for a class of states whose non-local character exceeds the bounds allowed by quantum mechanics. Motivated by our observation that an extension of the Schrödinger equation with nonlinear terms is directly linked to a relaxation of Born's rule, an axiom of quantum mechanics, we derive a physical theory that accounts for such hyper-correlated states and modifies Born's rule. We model correlated particles with a generalized probability theory whose dynamics are described with a non-linear version of Schrödinger's equation and demonstrate how that deviates from the standard formulation of quantum mechanics in experimental probability-prediction. We show also that the violation of the Clauser-Horn-Shimony-Holt inequality, the amount of non-locality, is proportional to the degree of non-linearity, which can be experimentally tested.
\end{abstract}

PACS numbers:

Physicists postulate the existence of a physical law that goes beyond quantum mechanics, which could lead to a modification of certain axioms underpinning quantum theory. The discovery of quantum mechanics at the dawn of the twentieth century led to major breakthroughs, from nuclear physics, microelectronics to quantum computing, which, by contrast to Newtonian physics, became known as modern physics. Quantum mechanics gives the most accurate description of microscopic objects like atoms and molecules.

In 1935, Einstein, Podolsky and Rosen, devised the eponymous EPR paradox [1], extended by John Bell in 1964 who formulated the Bell inequality which is violated by quantum mechanics. Their work demonstrated that quantum mechanics is nonlocal, such that quantum particles with correlated physical properties are entangled, or hyper-connected, irrespective of their physical location in space - a counter-intuitive notion that Einstein described as spooky action at a distance. The fundamentally probabilistic character of quantum mechanics means that non-locality does not make possible superluminal signalling, a causal constraint imposed by special relativity.

Bell's inequality provides a useful framework for quantifying non-locality of correlated physical properties. Above a threshold value of 2 , correlations violate the local realistic picture of classical physics. The value gives a measure of the amount of non-locality in the correlated quantum states, and can increase up to a maximum value of $2 \sqrt{2}$, called the Tsirelsôn bound [2], which characterises maximally entangled quantum states (or Bell states) under optimal measurement conditions. The bound sets a maximum amount of nonlocal correlations

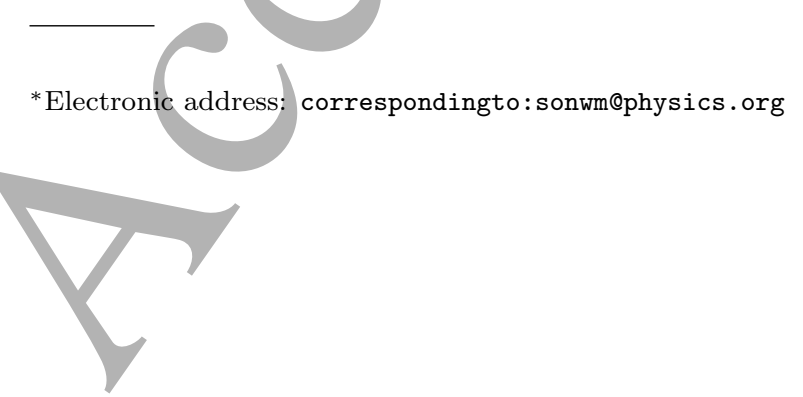

that quantum mechanics can allow or account for under the current axioms and, in particular, under the Born rule We lack a physical theory that describes what happens above this bound where the Bell inequality exceeds $2 \sqrt{2}$ and encompasses hyper-correlations that are stronger than nature allows under current theory of quantum mechanics. What we do know is that the nosignalling constraint fixes an absolute limit of 4 on the highest amount of non-local correlations permitted such that causality is not violated. An astute scenario, known as a Popescu-Rohrlich Box (PR Box) [3] (see also $[4,5]$ ) imagines how two quantum-entangled particles can in principle violate Bell's inequality such that the violation reaches a value of 4 .

The question, then, is what physical theory can account for the hyper-correlated systems between the Tsirelson and the PR Box bounds, and importantly, with what effect on the axioms underpinning quantum mechanics. This investigation provides an answer to this question in parallel with the previous discussions that can be found at $[6-9]$.

We start with the intuition that the physical theory is characterised by a non-linear dynamic equation. Previous extensions of non-linear quantum dynamics have been motivated by a need to describe wave-function collapse induced by gravity[10] based on the fact that we never observe a macroscopic object (say on Planck's mass scale or larger) in a superposition of separate and spatially distinct (say by a meter) states. The GhirardiRimini-Weber (GRW) non-linearity [11] was introduced to simulate such an objective collapse of the wave function, albeit its precise mechanism was left as an open problem.

Various gravitational theories that lead to apparently similar non-linearities such as they appear in the GRW model $[12,13]$ have been put forward. These are understood to be effective (i.e. non-fundamental) non- 


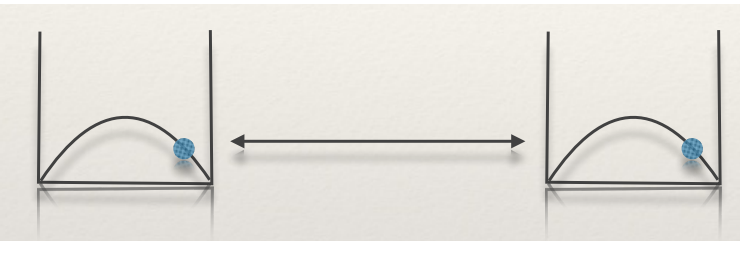

FIG. 1: Correlated particles confined in one dimensional box potentials

linearities, such as in the Gross-Pitaevski (GP) equation $[14,15]$, that arises merely out of the mean-field approximation applied to the linear many-body equation. In such a case, the non-linearity is a derived quantity and has no fundamental consequence. Another type of non-linearity called the log non-linearity, arises from the constraint of locality, has been put forward under the Bialynicki-Birula-Mycielski (BBM) scheme [16], showing that it satisfies the condition of separability between noninteracting sub-systems. Using the non-linear interaction, it proves that an isolated subsystem does not influence the physical properties of other subsystems. An experimental test of the log non-linearity in a neutron interferometer showed that the effect is not extensive [17].

Here, we demonstrate the consequences of a non-linear Schrödinger equation within generalised theories, resulting in different degrees of non-locality and a modification of a basic axiom in quantum theory. We assume that the non-linearity would not be a fundamental property inherent to the theory if it were induced by coupling to some external field such as gravity. The mechanism giving rise to the non-linearity is not of immediate concern. We demonstrate that the modified non-linear dynamic equation allows for a more general description of probabilities compared to the standard linear Schrödinger equation. The usefulness of the modified equation is illustrated by mapping it to Bell violations.

We show also that the non-linearity in the dynamic equation modifies Born's rule in standard quantum theory. The rule, we find, is surprisingly not dynamical in origin, but arises instead from the time-independent nonlinear Schrödinger equation.

The result gives a framework for realising a non-local box in the laboratory, while the parameter for the nonlinearity yields the estimated precision with which a quantum mechanical prediction can be considered accurate in current quantum theory.

First, we introduce briefly Bell's inequalities in the local hidden variable model. Quantum correlations are the characteristic trait of a quantum state that discriminate it categorically from any classical state [1], and provide also a resource for quantum information processing [18]. The non-local character of a quantum system characterised by the quantum correlations described by Bell [19] and Clauser-Horne-Shimony-Holt (CHSH) [20] still complies with Special Relativity. The Bell-CHSH func- tion is given by

$$
\mathcal{B}=E(\vec{a}, \vec{b})+E\left(\vec{a}, \vec{b}^{\prime}\right)+E\left(\vec{a}^{\prime}, \vec{b}\right)-E\left(\vec{a}^{\prime}, \vec{b}^{\prime}\right)
$$

where $E(\vec{a}, \vec{b})$ is a correlation function between two parties. In the local realistic (LR) model, the strict bound of the function is found as $|\mathcal{B}| \leq 2$ for the two measurement outcomes. Statistically speaking, the model imposes a strong constraint on the joint probabilities given by the two classical dichotomic systems.

Contrary to local realistic predictions, the bound can be violated by a quantum-correlated spin- $1 / 2$ system. For a quantum system, $E_{\text {quant }}(\vec{a}, \vec{b})$ is given as the amount of correlation under local measurements, parametrized by the three dimensional unit vectors $\vec{a}$ and $\vec{b}$ at each site. Through an idealised quantification using optimized measurement, the value becomes $E_{\text {quant }}(\vec{a}, \vec{b})=-\vec{a} \cdot \vec{b}=-\cos \left(\theta_{a b}\right)$ for the maximally entangled state e.g. a singlet state. It can be shown that the maximal value of $\mathcal{B}$ reaches $2 \sqrt{2}$, the Tsirelson bound. The physical origin of the maximal bound has been widely discussed, see e.g.[21], with certain theories predicting that the highest value of the Bell correlation can reach a value of 4 , without contradicting causality.

We present now our main result in relation to Bell correlations within the generalized-probability framework and the impact of the non-linear Schrödinger equation on Born's rule [22]. We consider with a non-linear equation (see below) where the single parameter potential of a system produces a function of particle density giving rise to the non-linearity. Once the eigenstates of the differential equation are identified, we show that the resulting probability does not coincide with that predicted by the modified Born's rule for the derived quantity. The solution to the non-linear dynamic equation yields a generalization of Born's rule that allows a calculation of Bell correlations in the non-linear regime. Consequently, any value of correlation between 2 and 4 can be attained, depending on the degree of non-linearity to which the dynamic system is subjected.

Let us consider a dynamic equation of two particle systems that are confined in a non-linear wave potential well as in Fig. 1. We assume that the dynamic equation in the coordinate system of relative motion is described by a Schrödinger-like wave equation,

$$
i \hbar \frac{\partial}{\partial t} \psi(x, t)=\left(-\frac{\hbar^{2}}{2 m} \frac{\partial^{2}}{\partial x^{2}}+g|\psi(x, t)|^{\epsilon}+V_{e x t}\right) \psi(x, t)
$$

where the parameter $x$ denotes the relative position of the particles which is $x=x_{2}-x_{1}$ for the individual 1 and 2 particles. Here, $\epsilon$ characterizes the amount of nonlinearity in the system. It is worth noting that it maps directly to the Bell correlation introduced by Popescu and Rohrlich[3] so does not violate the no-signalling constraint hence causality.

When $\epsilon=2$, the equation becomes a simplified version of the non-linear Schrödinger equation describing the 
density function of $N$ interacting Bosonic particles, called the Gross-Pitowsky (GP) equation [14, 15]. GP theory is a microscopic theory that describes an interacting nonuniform Bose gas at zero temperature see e.g.[23]. In that case, $g=4 \pi \hbar^{2} a / m$ characterizes the particle interactions and is defined in terms of the ground-state scattering length $a$. The normalization for $\psi$ is $N=\int|\psi|^{2} d^{3} \vec{r}$ and the potential can be the function of parameters for the displacement.

It can also describe non-equilibrium dynamics in cosmic structure and superfluid helium, called the KibbleZurek mechanism [24, 25]. From the perspective of ordinary partial differential equations, it is just a second order differential equation (ODE) with two parameters, spatial position $x$ and time $t$. In the context of the nonlinear Schrödinger equation, the GP equation describes also the evolution of a particle in a large gravitational field, as is called Diosi-Penrose (DP) equation. The DP mechanism has been used to describe wave function collapse due to the gravitational field $[12,13]$.

Our model provides a fundamentally different picture of nature than can be derived from standard quantum mechanics, or from exploring the physical consequences of models driven by an external non-linear effect such as gravity.

To simplify matters, we consider a solution of the free evolution $\psi(x, t)=\phi(x) e^{-i \omega t}$ whose external potential matches the resonant frequency, $V_{\text {ext }}=\hbar \omega$. In comparison to the original differential equation, the non-linear Schrödinger (NS) equation can take the form

$$
\frac{\hbar^{2}}{2 m} \frac{\partial^{2} \phi(x)}{\partial x^{2}}+\bar{g}|\phi(x)|^{\epsilon} \phi(x)=0
$$

where we consider the case, $\bar{g}=-g$. The negative $g$ may not be physically relevant in a real situation/but here we assume the property for analytic purposes. With these parameters, the solution of the static NS equation can be found analytically such that

$$
\phi_{n, \alpha}(x)=\frac{\alpha T}{n \pi_{2,2+\epsilon}} \sin _{2,2+\epsilon}\left(n \pi_{2,2+\epsilon} x\right)
$$

where the positive constant of the coefficient takes the values $m \bar{g} / \hbar=\left(n \pi_{2,2+\epsilon}\right)^{4} /|\alpha|^{2}$. Here, the special function $\sin _{2,2+\epsilon}(\cdot)$ denotes the generalized trigonometric function which is obtained in the work of Lundberg [26] (see also[27]), Levin [28] and [29, 30] as an analytical periodic function. The analytic form of the function is possible to be obtained as

$$
\sin _{2,2+\epsilon}(\theta)=F_{2,2+\epsilon}^{-1}(\theta)
$$

where $F_{p, q}(\theta)=\int_{0}^{\theta}\left(1-t^{q}\right)^{-1 / p} d t$ and $\pi_{p, q}:=2 F_{p, q}(1)$. $\pi_{p, q}=2 \Gamma(1-1 / p) \Gamma(1 / q) / q \Gamma(1-1 / p+1 / q)$ with gamma function $\Gamma, \pi_{p}=\pi_{p, p}=2 \pi /[p \sin (\pi / p)]$ and $\pi_{2}=\pi$. The function $\sin _{p, q}(\theta)$ is a well-behaving periodic function, which is analytic within the period and whose periodicity is given by a newly defined irrational number $\pi_{p, q}$ that becomes ordinary $\pi$ when $\pi_{2,2}=\pi$.
Then the spacial part of the function $\phi(x)$ can be interpreted as a wave-function-like object so far as it gives the coincident probabilities of a spin measurement on the correlated system, but whose physical properties in general differ from those of a wave function. The statement can be expressed by the equalities

$$
p(a=b)=p(\uparrow \uparrow)+p(\downarrow \downarrow)=|\phi(x)|^{2}
$$

giving the probability of symmetric coincident events. Without a detailed description of the local wave function for individual particles, the function $\phi(x)$ represents the probability density of the composite system, equivalent to "clicks" in detectors triggered by coincident particles. The only relevant external parameter for the function in this case is the relative displacement corresponding to the choice of measurement settings through the adjusted external parameters. At this generalised level, we describe only the probability of events (e.g. coincidence counts) and no longer consider state preparation and measurements on individual states.

The existence of the probability function also means that there is a conjugate probability and its corresponding wave function. The function can be derived from the first derivative of the original wave function whose explicit form can be obtained analytically as $\cos _{2,2+\epsilon}(x)=$ $\frac{\partial}{\partial x} \sin _{2,2+\epsilon}(x)=\phi(x)$. Similarly, the conjugate probability of the non-linear Schrödinger equation can be written as

$$
p(a \neq b)=p(\uparrow \downarrow)+p(\uparrow \downarrow)=|\bar{\phi}(x)|^{2+\epsilon}
$$

in order to satisfy the normalization condition. It follows from the constraint that the normalization condition of the probability is

$$
|\phi(x)|^{2}+|\bar{\phi}(x)|^{2+\epsilon}=1
$$

since it satisfies the condition of the generalized trigonometric function and $p(a=b)+p(a \neq b)=1$. It implies that the solution of the wave equation deviates from Born's rule by $\epsilon$ when one tries to obtain the conjugate probability from the solution of the non-linear equation $\phi(x)$. Next, we dicsuss how that a deviation from Born's rule violates Bell's inequalities when we go beyond the Tsirelson bound.

In the Bell-CHSH inequality, correlation functions with different choices of measurements on the particles need to be identified. They can be obtained from the solution of the non-linear dynamic equation as

$$
E_{\epsilon}(\theta)=p(a=b)-p(a \neq b)=|\phi(\theta)|^{2}-|\bar{\phi}(\theta)|^{2+\epsilon}
$$

where $\phi$ and $\bar{\phi}$ are the probability densities for the measurement probabilities. The second equation uses the normalization condition $|\phi(\theta)|^{2}+|\bar{\phi}(\theta)|^{2+\epsilon}=1$. It means also that the correlation function is subject to singlevalue parametrization whose physical meaning relates directly to the angle between the local measurement at

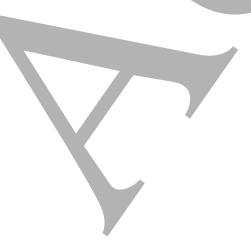


stations A and B, such as phase, amplitude, position or momentum.

In that virtual scenario, the wave-function-like object $\phi(\theta)$ can be related to physical quantities, such as the total particle number of the systems, where $N=$ $\int|\phi(\theta)|^{2} d \theta$. Additionally, its conjugate function becomes $\bar{N}=\int|\bar{\phi}(\theta)|^{2+\epsilon} d \theta$. An additional degree of freedom, such as a spin-like property, can be added to the function by indexing it to a measurable quantity. The symmetry condition for our wave function is fulfilled by our choice of the variable, theta, which is deliberately chosen to be symmetric, such that its indices remain unchanged under exchange.

Interestingly, one can show that the Bell-CHSH inequality is violated above the Tsirelson bound using the modified correlation function. The Bell function in (1), after non-linear modification, becomes

$$
\mathcal{B}_{\epsilon}=E_{\epsilon}\left(\theta_{a b}\right)+E_{\epsilon}\left(\theta_{a b^{\prime}}\right)+E_{\epsilon}\left(\theta_{a^{\prime} b}\right)-E_{\epsilon}\left(\theta_{a^{\prime} b^{\prime}}\right)
$$

where $E_{\epsilon}(\theta)$ is the same function as in (9). When the Bell function becomes larger than 2, the system cannot be explained by a local realistic model, hence we need a theory that goes beyond that. Fig. 2 shows the optimized value of $\mathcal{B}_{\epsilon}$ with respect to $\epsilon$. To obtain the maximum value of the Bell function, a linear optimization over the parameters $\left(\theta_{a b}, \theta_{a b^{\prime}}, \theta_{a^{\prime} b}, \theta_{a^{\prime} b^{\prime}}\right)$ was carried out, represented by the blue curve in Fig. 2. $\mathcal{B}_{\epsilon}$ can be in the range of $2<\mathcal{B}_{\epsilon}<4$ as it is monotonically varied, and tells us that the Bell-CHSH correlation can take a value beyond the local realistic model and current quantum theory, depending on the parameter $\epsilon$.

The behaviour can be extrapolated from the optimised plot of the Bell-CHSH function and a single parameterized Ansatz of a simplified distribution is given by

$$
\max \left[\mathcal{B}_{\epsilon}\right]=4 / 2^{(1+\epsilon) /(2+\epsilon)} .
$$

We note that its value becomes 2 for $\epsilon \rightarrow \infty, 2 \sqrt{2}$ for $\epsilon=0$ and 4 for $\epsilon \rightarrow-1$, as can be seen in Fig. 2 and in the inset for a marginal error fit for comparison. The functional behaviour follows the original Bell function to within a $10 \%$ deviation.

We can clearly see that the Bell violation can go above the Tsirelson bound when the non-linear parameter $\epsilon$ takes a negative value. That is the case when the nonlinear term in the dynamic equation becomes inversely proportional to the wave function as the term is asymptotically amplified in the limit $\epsilon \rightarrow-1$. It is highly instructive to see that its value covers the entire range from the local hidden variable model to the maximum value within causality.

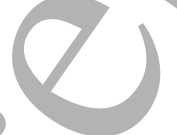

[1] A. Einstein, B. Podolsky and N. Rosen, Phys. Rev. Lett. 47, 777 (1935).

[2] B. S. Tsirelson, Lett. Math. Phys. 4, 93 (1980).
In conclusion, we have demonstrated a model of correlated particles whose dynamics are subject to a non-linear Schrödinger equation and the consequences of the model.

An analytic solution of the non-linear dynamic equation is given by the Dirichlet eigenvalue problem, and consequently, a deviation from quantum theory in the probability prediction of laboratory experiments, is demonstrated.

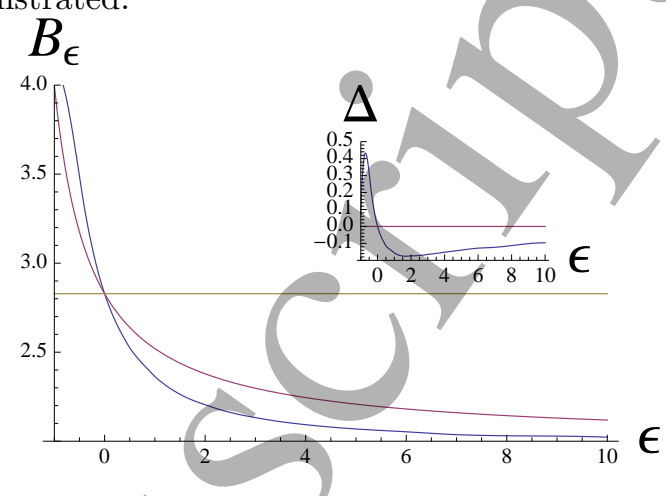

FIG. 2: Degree of Bell violation $\mathcal{B}_{\epsilon}$ for different values of $\epsilon$. It varies continuously between $2<\mathcal{B}_{\epsilon}<4$ and is generated for $-1<\epsilon<\infty$ in the modified Born's rule. The numerical value of the Bell violation agrees with the symmetric value of the Bell correlation. The blue line corresponds to the value obtained by numerical simulation. The red line plots the function from our symmetric conjecture.

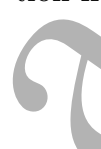

A modified formulation of Born's rule has been obtained by relating the event probability of measurement to an arbitrary exponent of the modulus of the eigenvalue solution, illustrating that the violation of the ClauserHorne-Shimony-Holt inequality is proportional to the degree of the non-linearity, which can be verified by Bell test experiments. Depending on the degree of non-linearity, the violation can go above the Tsirelson bound $2 \sqrt{2}$ and reach the value of a nonlocal box for hyper-correlations beyond quantum mechanics.

Acknowledgements- TF and VV acknowledge support from the National Research Foundation and the Ministry of Education administered by Centre for Quantum Technologies, National University of Singapore. WS acknowledges support from Korean Institute of Advanced Study, Samsung and KRISS. WS conceived the idea and obtained the relevant results. TF and WS formulated the concept and wrote the manuscript. WS and VV discussed the interpretation of the results extensively.

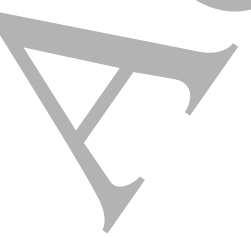

[3] S. Popescu and D. Rohrlich, Found. Phys. 24, 379 (1994).

[4] S. Popescu, Nature Physics 10, 264 (2014).

[5] V. Scarani, Bell Nonlocality (OUP, 2019). 
[6] Y. Aharonov, unpublished lecture note; see also, Y. Aharonov and D. Rohrlich, Quantum Paradoxes(WILEY-VCH, 2005).

[7] W. van Dam, Ph.D. Thesis, University of Oxford (2000), quant-ph/0501159.

[8] G. Brassard, H. Buhrman, N. Linden, A. Methot, A. Tapp and F. Unger, Phys. Rev. Lett. 96, 250401 (2006).

[9] J. Barrett, Phys. Rev. A 75, 032304 (2007).

[10] A. Bassi, K. Lochan, S. Satin, T. P. Singh and H. Ulbricht, Rev. Mod. Phys. 85, 471 (2013).

[11] G. C. Ghirardi, A. Rimini, and T. Weber, Phys. Lett. D 34470 (1986)

[12] L. Diósi, Phys. Lett. A 120, 377 (1987).

[13] R. Penrose, Gen. Relativ. Gravit. 28, 581 (1996).

[14] E. P. Gross (May 1961). "Structure of a quantized vortex in boson systems". Il Nuovo Cimento 20 (3): 454-457

[15] L. P. Pitaevskii, Soviet Physics JETP 13 (2): 451 (1961).

[16] Bialynicki-Birula, I., Mycielski, J., Ann. Phys. (N.Y.), 100, 62 (1976)

[17] Gähler, Klein A.G., Zeilinger A., Neutron optical tests of nonlinear wave mechanics, Phys. Rev. A, 23, 1611 (1981).

[18] M. A. Nielsen and I. L. Chuang, Quantum Computation and Quantum Information (Cambridge University Press, Cambridge, 2000).

[19] J. S. Bell, Physics 1, 195 (1964).

[20] J. F. Clauser, M.A. Horne, A. Shimony and R. A. Holt, Phys. Rev. Lett. 23, 880 (1969).

[21] M. Epping, H. Kampermann, and D. Bruss Phys. Rev. Lett. 111, 240404 (2013).

[22] M. Born, Zur Quantenmechanik der Stoßvorgänge. Z. Physik 37, 863-867 (1926)

[23] A. Leggett, Rev. Mod. Phys., 73:307-356, (2001)

[24] T. W. B. Kibble, J. Phys. A: Math. Gen. 9: 1387 (1976).

[25] W. H. Zurek, Nature 317: 505 (1985)

[26] E. Lundberg, Om hypergoniometriska funktioner af komplexa variabla, Stockholm, 1879. English translation: On hypergoniometric functions of complex variables.

[27] P. Lindqvist, Ricerch Mat. 44 (2) 269-290 (1995).

[28] V.I. Levin, Rec. Math. Moscou, 4, 309-331 (1938).

[29] D.E. Edmunds, P. Gurka, J. Lang, Journal of Approximation Theory, 164, 47-56 (2012).

[30] P. Drabek and R. Manasevich, Differential and Integral Equations. 12, 773(1999). 